\title{
COUNTEREXAMPLES RELATED TO COMMUTATORS OF UNBOUNDED OPERATORS
}

\author{
MOHAMMED HICHEM MORTAD
}

\begin{abstract}
The present paper is exclusively devoted to counterexamples about commutators and self commutators of unbounded operators on a Hilbert space. As a bonus, we provide a simpler counterexample than McIntosh's famous example obtained some while ago.
\end{abstract}

\section{INTRODUCTION}

The formal commutator of two non necessarily bounded operators $A$ and $B$ is defined to be $A B-B A$. We have called it "formal" as, unlike the bounded case, $A B-B A=0$ does not always imply the (strong) commutativity of $A$ and $B$ when say $A$ and $B$ are self-adjoint. The first such counterexample is due to Nelson [10. Apparently, the first textbook to include it is [13]. The same example is developed in detail in [14, pp 257-258. Perhaps the simplest counterexample is due to Schmüdgen and may be found in e.g. [15].

The self commutator of a densely defined operator $T$ is defined to be $T T^{*}-T^{*} T$.

The main purpose of this paper is to exhibit counterexamples to questions related to commutators and self-commutators as regards boundedness, closedness and selfadjointness.

Readers throughout the paper will observe how fascinating the use of matrices of unbounded operators helps to find such counterexamples. The same approach has equally allowed us to find more interesting counterexamples on a different topic. See [9]. Readers should be wary that it took me some time before I came up with these relatively simple examples.

We refer readers to [16] for properties and results about matrices of unbounded operators. See also [8] or [11. For the general theory of unbounded operators, readers may wish to consult [15] or [17]. See also [12]. Finally, it is worth noticing that there is an extensive work on estimating the norm of commutators of some classes of bounded operators. We cite among others: [2], [3, [4] and [5].

\section{Main Counterexamples}

We start with an auxiliary result which is also interesting in its own.

Proposition 2.1. There exists a densely defined unbounded and closed operator $B$ such that $B^{2}$ and $|B| B$ are bounded whereas $B|B|$ is unbounded and closed.

Proof. Let $H$ be a complex Hilbert space and let $A$ be an unbounded self-adjoint and positive operator with domain $D(A) \subset H$ (for instance $A f(x)=e^{x^{2}} f(x)$ on its

2010 Mathematics Subject Classification. Primary 47B47. Secondary 47A05, 47B25.

Key words and phrases. Commutators. Self-commutators. Bounded and Unbounded Operators. 
maximal domain on $\left.L^{2}(\mathbb{R})\right)$. Let

$$
B=\left(\begin{array}{cc}
0 & A \\
0 & 0
\end{array}\right)
$$

be defined on $H \oplus D(A)$. Then $B$ is closed and as seen before $B^{2}=0$ on $H \oplus D(A)$. Now,

$$
|B|=\left(\begin{array}{cc}
0 & 0 \\
0 & A
\end{array}\right) \text { and so }|B| B=\left(\begin{array}{cc}
0 & 0_{D(A)} \\
0 & 0_{D(A)}
\end{array}\right),
$$

that is, $|B| B$ is bounded on $H \oplus D(A)$. However,

$$
B|B|=\left(\begin{array}{cc}
0 & A^{2} \\
0 & 0
\end{array}\right)
$$

is clearly unbounded on $H \oplus D\left(A^{2}\right)$.

Proposition 2.2. There are two densely defined unbounded and closed operators $B$ and $C$ such that $C B-B C$ is bounded and unclosed while $|C| B-B|C|$ is unbounded and closed.

Proof. Let $B$ be as in Proposition 2.1 and set $C=B$. Then $C B-B C=0_{D\left(B^{2}\right)}$ is clearly bounded and unclosed. By a glance at Proposition 2.1 again, we easily see that

$$
|B| B-B|B|=\left(\begin{array}{cc}
0 & A^{2} \\
0 & 0
\end{array}\right)
$$

and that it is closed (and unbounded) on $D(|B| B-B|B|)=H \oplus D\left(A^{2}\right)$.

Lemma 2.3. There are two unbounded and self-adjoint operators $A$ and $B$ such that $D(A)=D(B), A^{2}-B^{2}$ is bounded but $A B-B A$ is unbounded.

Proof. Let $T$ be any unbounded and self-adjoint operator with domain $D(T) \subset H$ where $H$ is a Hilbert space. Next, define $A=\left(\begin{array}{cc}0 & T \\ T & 0\end{array}\right)$ and $B=\left(\begin{array}{cc}T & 0 \\ 0 & -T\end{array}\right)$ and so $D(A)=D(B)=D(T) \oplus D(T)$. Hence $A^{2}=B^{2}=\left(\begin{array}{cc}T^{2} & 0 \\ 0 & T^{2}\end{array}\right)$ and so $A^{2}-B^{2}$ is bounded (on $D\left(A^{2}\right)$ ) whilst

$$
A B-B A=\left(\begin{array}{cc}
0 & -2 T^{2} \\
2 T^{2} & 0
\end{array}\right)
$$

is obviously unbounded.

We know that there exist two unbounded self-adjoint operators $A$ and $B$ such that $A B-B A$ is bounded (on its domain) while $|A| B-B|A|$ is unbounded. The first (and apparently the only) counterexample is due to McIntosh in [7] who answered a question raised by the great T. Kato. The example we are about to give here is new and simpler than McIntosh's. Moreover, in our case both $A B-B A$ and $|A| B-B|A|$ are even closed.

Proposition 2.4. There exist two unbounded and self-adjoint operators $A$ and $B$ such that $A B-B A$ is bounded (and closed) whilst $|A| B-B|A|$ is unbounded (and also closed).

The counterexample is based on the following recently obtained result: 
Lemma 2.5. (1]) There are unbounded self-adjoint operators $A$ and $B$ such that

$$
D\left(A^{-1} B\right)=D\left(B A^{-1}\right)=\{0\}
$$

(where $A^{-1}$ and $B^{-1}$ are not bounded).

Now, we give the promised counterexample:

Proof. Let $R, S, T$ be three self-adjoint operators on a Hilbert space $H$ with domains $D(R), D(S)$ and $D(T)$ respectively. Assume also that $S$ is positive. Now, define on $H \oplus H$ the operators

$$
A=\left(\begin{array}{cc}
0 & S \\
S & 0
\end{array}\right) \text { and } B=\left(\begin{array}{cc}
T & 0 \\
0 & R
\end{array}\right)
$$

with domains $D(A)=D(S) \oplus D(S)$ and $D(B)=D(T) \oplus D(R)$ respectively. Hence

$$
A B-B A=\left(\begin{array}{cc}
0 & S R-T S \\
S T-R S & 0
\end{array}\right) .
$$

Since $|A|=\left(\begin{array}{cc}S & 0 \\ 0 & S\end{array}\right)$, it follows that

$$
|A| B-B|A|=\left(\begin{array}{cc}
S T-T S & 0 \\
0 & S R-R S
\end{array}\right) .
$$

To obtain the appropriate operators, let $C$ and $D$ be such that $D(C D)=$ $D(D C)=\{0\}$ (as in Lemma 2.5). Remember that $C$ is self-adjoint, positive, unbounded and (not boundedly) invertible. Define now

$$
S=\left(\begin{array}{cc}
0 & C \\
C & 0
\end{array}\right) \text { and } T=\left(\begin{array}{cc}
D & 0 \\
0 & D
\end{array}\right)
$$

and so

$$
S T=\left(\begin{array}{cc}
0 & C D \\
C D & 0
\end{array}\right) \text { and } T S=\left(\begin{array}{cc}
0 & D C \\
D C & 0
\end{array}\right) .
$$

Hence $D(S T)=D(T S)=\left\{0_{\left[L^{2}(\mathbb{R})\right]^{2}}\right\}$. This says that $A B-B A$ is bounded on $L^{2}(\mathbb{R}) \oplus L^{2}(\mathbb{R}) \oplus L^{2}(\mathbb{R}) \oplus L^{2}(\mathbb{R})$. In fact, $A B-B A$ is trivially bounded as it is only defined on $\{0\}$ and $A B-B A$ is therefore closed. In order that $|A| B-B|A|$ be unbounded, it suffices then to exhibit a self-adjoint and unbounded $R$ such that $S R-R S$ is unbounded. Consider $R=\left(\begin{array}{cc}C^{-\frac{1}{2}} & 0 \\ 0 & C\end{array}\right)$. Therefore,

$$
S R=\left(\begin{array}{cc}
0 & C^{2} \\
\sqrt{C} & 0
\end{array}\right), R S=\left(\begin{array}{cc}
0 & \sqrt{C} \\
C^{2} & 0
\end{array}\right)
$$

and

$$
S R-R S=\left(\begin{array}{cc}
0 & C^{2}-\sqrt{C} \\
\sqrt{C}-C^{2} & 0
\end{array}\right) .
$$

Finally, $C^{2}-\sqrt{C}$ is unbounded (and self-adjoint). Indeed, since $C$ is self-adjoint, it is unitarily equivalent to the multiplication operator $M_{\varphi}$ by an unbounded and real-valued (positive) function $\varphi$. Hence, $C^{2}-\sqrt{C}$ is unitarily equivalent to the multiplication operator by the unbounded real-valued $\varphi^{2}-\sqrt{\varphi}$. Thus, as $C^{2}-\sqrt{C}$ is unbounded, $S R-R S$ too is unbounded and so $|A| B-B|A|$ is equally unbounded (and closed), as coveted. 
Now, we consider the case of self-commutators. First, we show that the selfcommutator of a densely defined and closed operator may only be defined at 0 .

Proposition 2.6. There exists a densely defined and closed operator $T$ such that $D\left(T T^{*}\right) \cap D\left(T T^{*}\right)=\{0\}$ and hence

$$
D\left(T T^{*}-T^{*} T\right)=\{0\} .
$$

Proof. Consider two unbounded and self-adjoint operators $A$ and $B$ which obey $D(A) \cap D(B)=\{0\}$ (see e.g. [6]). Set $T=\left(\begin{array}{cc}0 & A \\ B & 0\end{array}\right)$ and so $T^{*}=\left(\begin{array}{cc}0 & B \\ A & 0\end{array}\right)$. Hence

$$
T T^{*}=\left(\begin{array}{cc}
A^{2} & 0 \\
0 & B^{2}
\end{array}\right) \text { and } T^{*} T=\left(\begin{array}{cc}
B^{2} & 0 \\
0 & A^{2}
\end{array}\right) \text {. }
$$

Therefore,

$$
D\left(T T^{*}\right) \cap D\left(T T^{*}\right)=\left[D\left(A^{2}\right) \cap D\left(B^{2}\right)\right] \oplus\left[D\left(B^{2}\right) \cap D\left(A^{2}\right)\right]
$$

and so

$$
D\left(T T^{*}\right) \cap D\left(T T^{*}\right) \subset[D(A) \cap D(B)] \oplus[D(B) \cap D(A)]=\{(0,0)\},
$$

as needed.

In the next two counterexamples, we show that $T T^{*}-T^{*} T$ may be bounded but $|T|\left|T^{*}\right|-\left|T^{*}\right||T|$ may be not, and vice versa.

Proposition 2.7. There exists a densely defined and closed operator $T$ such that $T T^{*}-T^{*} T$ is unbounded but $|T|\left|T^{*}\right|-\left|T^{*}\right||T|$ is bounded (even zero on some domain!).

Proof. Let $A$ be an unbounded, self-adjoint and positive operator with domain $D(A) \subset H$. Define $T$ with domain $D(T)=D(A) \oplus H$ by

$$
T=\left(\begin{array}{cc}
0 & 0 \\
A & 0
\end{array}\right) \text { and so } T^{*}=\left(\begin{array}{cc}
0 & A \\
0 & 0
\end{array}\right) .
$$

Hence

$$
T T^{*}=\left(\begin{array}{cc}
0 & 0 \\
0 & A^{2}
\end{array}\right) \text { and } T^{*} T=\left(\begin{array}{cc}
A^{2} & 0 \\
0 & 0
\end{array}\right)
$$

Also,

$$
|T|\left|T^{*}\right|=\left(\begin{array}{cc}
0 & 0_{D(A)} \\
0 & 0_{D(A)}
\end{array}\right) \text { and }\left|T^{*}\right||T|=\left(\begin{array}{cc}
0_{D(A)} & 0 \\
0_{D(A)} & 0
\end{array}\right) .
$$

Thus,

$$
T T^{*}-T^{*} T=\left(\begin{array}{cc}
-A^{2} & 0 \\
0 & A^{2}
\end{array}\right)
$$

is clearly unbounded (and self-adjoint) whereas $|T|\left|T^{*}\right|-\left|T^{*}\right||T|$ is bounded (in fact, it is the zero operator on $D(A) \oplus D(A))$.

Proposition 2.8. There exists a densely defined and closed operator $T$ such that $T T^{*}-T^{*} T$ is bounded whereas $|T|\left|T^{*}\right|-\left|T^{*}\right||T|$ is unbounded. 
Proof. Let $A$ and $B$ be two self-adjoint and positive operators with domains $D(A)$ and $D(B)$. Set $T=\left(\begin{array}{cc}0 & \sqrt{A} \\ \sqrt{B} & 0\end{array}\right)$. Hence

$$
T T^{*}-T^{*} T=\left(\begin{array}{cc}
A-B & 0 \\
0 & B-A
\end{array}\right)
$$

and

$$
|T|\left|T^{*}\right|-\left|T^{*}\right||T|=\left(\begin{array}{cc}
\sqrt{B} \sqrt{A}-\sqrt{A} \sqrt{B} & 0 \\
0 & \sqrt{A} \sqrt{B}-\sqrt{B} \sqrt{A}
\end{array}\right) .
$$

To get the desired counterexample, it suffices to have $D(A) \cap D(B)=\{0\}$ and so $T T^{*}-T^{*} T$ becomes trivially bounded, and at the same time, the same pair must make $\sqrt{A} \sqrt{B}-\sqrt{B} \sqrt{A}$ unbounded (and thus $|T|\left|T^{*}\right|-\left|T^{*}\right||T|$ too becomes unbounded). One possible choice is to consider $\sqrt{A}$ to be defined by

$$
\sqrt{A} f(x)=e^{\frac{x^{2}}{4}} f(x)
$$

on $D(\sqrt{A})=\left\{f \in L^{2}(\mathbb{R}): e^{\frac{x^{2}}{4}} f \in L^{2}(\mathbb{R})\right\}$. Then $\sqrt{A}$ is self-adjoint, positive and boundedly invertible. Set $\sqrt{B}:=\mathcal{F}^{*} \sqrt{A} \mathcal{F}$ where $\mathcal{F}$ denotes the usual $L^{2}(\mathbb{R})$ Fourier Transform. Then $\sqrt{B}$ too is self-adjoint, positive and boundedly invertible. We know that $D(A) \cap D(B)=\{0\}$ (see e.g. 6]). So, it only remains to check that $\sqrt{B} \sqrt{A}-\sqrt{A} \sqrt{B}$ is in unbounded. A way of seeing that is to consider the action of this operator on some sequence of Gaussian functions. This finishes the proof.

\section{Conjecture}

Inspired by the pair $S$ and $R$ which appeared in Proposition 2.4, we propose the following conjecture:

Conjecture 2.9. For any unbounded self-adjoint operator $A$, there is always a bounded and self-adjoint operator $B$ such that $A B-B A$ is unbounded.

\section{REFERENCES}

[1] S. Dehimi, M. H. Mortad, Chernoff Like Counterexamples Related to Unbounded Operators, (submitted). arXiv:1808.09523 2 .

[2] C. K. Fong, Norm estimates related to self-commutators, Linear Algebra Appl., 74 (1986) 151-156.

[3] F. Kittaneh, Inequalities for commutators of positive operators, J. Funct. Anal., 250/1 (2007) 132-143.

[4] F. Kittaneh, Norm inequalities for commutators of positive operators and applications, Math. Z., 258/4 (2008), 845-849.

[5] F. Kittaneh, Norm inequalities for commutators of self-adjoint operators, Integral Equations Operator Theory, 62/1 (2008) 129-135.

[6] H. Kosaki, On intersections of domains of unbounded positive operators, Kyushu J. Math., 60/1 (2006) 3-25.

[7] A. McIntosh, Counterexample to a question on commutators, Proc. Amer. Math. Soc., 29 (1971) 337-340.

[8] M. Möller, F. H. Szafraniec, Adjoints and Formal Adjoints of Matrices of Unbounded Operators, Proc. Amer. Math. Soc., 136/6 (2008) 2165-2176.

[9] M. H. Mortad, On the triviality of domains of powers and adjoints of closed operators.

[10] E. Nelson, Analytic vectors, Ann. of Math. (2), 70 (1959) 572-615.

[11] S. Ôta, K. Schmüdgen, Some selfadjoint $2 \times 2$ operator matrices associated with closed operators, Integral Equations Operator Theory, 45/4 (2003) 475-484.

[12] C. R. Putnam, Commutation properties of Hilbert space operators and related topics, Springer-Verlag, New York, 1967. 
[13] M. Reed, B. Simon, Methods of Modern Mathematical Physics, Vol. 1: Functional Analysis, Academic Press, 1972.

[14] K. Schmüdgen, Unbounded operator algebras and representation theory. Operator Theory: Advances and Applications, 37. Birkhäuser Verlag, Basel, 1990.

[15] K. Schmüdgen, Unbounded Self-adjoint Operators on Hilbert Space, Springer. GTM 265 (2012).

[16] Ch. Tretter, Spectral Theory of Block Operator Matrices and Applications. Imperial College Press, London, 2008.

[17] J. Weidmann, Linear Operators in Hilbert Spaces, Springer, 1980.

Department of Mathematics, University of Oran 1, Ahmed Ben Bella, B.P. 1524, El Menouar, Oran 31000, Algeria.

Preferred mailing address:

Pr Mohammed Hichem Mortad

BP 7085 Seddikia Oran

31013

Algeria

E-mail address: mhmortad@gmail.com, mortad.hichem@univ-oran1.dz. 